\title{
Multi-Objective Structural Design Optimization using Fuzzy Optimization Programming based on T-Norm
}

\author{
Samir Dey \\ Department of Mathematics Asansol Engineering \\ College \\ Vivekananda Sarani, \\ Asansol-713305, West Bengal, India
}

\author{
Tapan Kumar Roy \\ Department of Mathematics \\ Indian Institute of science Engineering and technology \\ P.O.Botanic Garden, \\ Howrah-711103, West Bengal, India
}

\begin{abstract}
In this paper we propose an approach to solve multi-objective structural design problem using basic t-norm based fuzzy optimization programming technique. Here a planer truss structural model in fuzzy environment has been developed. In this structural model formulation, the objective functions are the weight of the truss and the vertical deflection of loaded joint; the design variables are the cross-sections of the truss members; the constraints are the stresses in members. A classical truss optimization example is presented here in to demonstrate the efficiency of our propose optimization approach. The test problem includes a three-bar planar truss subjected to a single load condition. This approximation approach is used to solve this multi-objective structural optimization model. The model is illustrated with numerical examples.
\end{abstract}

\section{Keywords}

Multi-objective Optimization, Triangular Norm, Fuzzy Set, Structural Optimization

\section{INTRODUCTION}

Optimization is the process of minimizing or maximizing an objective function (e.g. cost, weight) of a structural system which has been frequently employed as the evaluation criterion in structural engineering applications. But in the practical optimization problems, usually more than one objective are required to be optimized, such as minimum mass or cost, maximum stiffness, minimum displacement at specific structural points, maximum natural frequency of free vibration, and maximum structural strain energy. This makes it necessary to formulate a multi-objective optimization problem. The first note on multi-objective optimization was given by Pareto; since then the determination of the compromise set of a multi-objective problem is called Pareto optimization. That is why the application of different optimization technique $[5,19,20-24]$ to structural problems has attracted the interest of many researchers.

In real life, the data cannot be recorded or collected precisely due to human errors or some unexpected situations. So one may consider ambiguous situations like vague parameters, non-exact objective and constraint functions in the problem and it may be classified as a non-stochastic imprecise model. Here fuzzy set theory may provide a method to describe or formulate this imprecise model. Zadeh [2] first gave the concept of fuzzy set theory for handling uncertainty that is due to imprecision rather than to randomness. Later on
Bellman and Zadeh [3] used the fuzzy set theory to the decision making problem. Zimmermann [4] proposed a fuzzy multi-criteria decision making set, defined as the intersection of all fuzzy goals and their constraints.

In practical, the problem of structural design may be formed as a typical non-linear programming problem with non-linear objective functions and constraints functions in fuzzy environment. Some researchers applied the fuzzy set theory to Structural model. For example Wang et al. [1] first applied $\alpha$-cut method to structural designs where the non-linear problems were solved with various design levels $\alpha$, and then a sequence of solutions are obtained by setting different levelcut value of $\alpha$. Rao [14] applied the same $\alpha$-cut method to design a four-bar mechanism for function generating problem .Structural optimization with fuzzy parameters was developed by Yeh et.al [7]. In 1989, Xu [6] used two-phase method for fuzzy optimization of structures. In 2004, Shih et.al [15] used level-cut approach of the first and second kind for structural design optimization problems with fuzzy resources .Shih et.al [16] develop an alternative $\alpha$-level-cuts methods for optimum structural design with fuzzy resources in 2003. Dey et.at [5] optimize multi-objective structural model with fuzzy coefficient i.e. generalized fuzzy number and its total $\lambda$ integral value.

Alsina et.al. [8] introduced the t-norm into fuzzy set theory and suggested that the t-norms be used for the intersection of fuzzy sets. Different types of t-norms theory and their fuzzy inference methods were introduced by Gupta et.al.[9] .The extension of fuzzy implication operators and generalized fuzzy methods of cases were discussed by Ruan et.al. [10] .Pei et.al [11] introduced the extended t-norms and another kind of fuzzy universal algebras. Kaymak et.al. [12] use weighted extension of (Archimedean) fuzzy t-norms in optimization of various criteria model. Samanta et.al.[13] solve portfolio selection model using extended t-norm based fuzzy optimization technique.

In this paper we propose an approach to solve multi-objective structural model using t-norms based fuzzy optimization programming technique. In this structural model formulation, the objective functions are the weight of the truss and the vertical deflection of loaded joint; the design variables are the cross-sections of the truss members; the constraints are the stresses in members. The test problem includes a three-bar planar truss subjected to a single load condition. This 
approximation approach is used to solve this multi-objective structural optimization model.

The remainder of this paper is organized in the following way. In section 2, we discuss about structural optimization model. In section 3, we discuss about mathematics Prerequisites. In section 4, we discuss about t-norm and extended n-ary tnorms. In section 5 , we discuss about the basic t-norm and their generalization with weight factors. In section 6, we discuss about weighted fuzzy aggregation. In section 7 , we proposed the technique to solve multi-objective non-linear programming problem using extended t-norm based fuzzy optimization. In section 8 , we solve multi-objective structural model using extended t-norms based fuzzy optimization. In section 9, numerical solution of structural model of three bar truss and compared results by using different extended weighted t-norms. Finally we draw conclusions in section 10 .

\section{MULTI-OBJECTIVE STRUCTURAL MODEL}

In the design of optimal structure i.e. lightest weight of the structure and minimum deflection of loaded joint that satisfies all stress constraints in members of the structure. To bar truss structure system the basic parameters (including the elastic modulus, material density, the maximum allowable stress, etc.) are known and the optimization's target is that identify the optimal bar truss cross-section area so that the structure is of the smallest total weight, the minimum nodes displacement, in a given load conditions.

The multi-objective Structural model can be expressed as

$$
\begin{gathered}
\text { minimize } W T(A) \\
\text { minimize } \delta(A) \\
\text { subject to } \quad \sigma(A) \leq\left[\sigma_{0}\right] \\
\qquad A_{\min } \leq A \leq A_{\max }
\end{gathered}
$$

where $A=\left[A_{1}, A_{2}, \ldots . ., A_{n}\right]^{T}$ are design variables for the cross section, $\mathrm{n}$ is the group number of design variables for the cross section bar, $W T=\sum_{i=1}^{n} \rho_{i} A_{i} L_{i}$ is the total weight of the structure, $\delta(A)$ is the deflection of loaded joint $L_{i}, A_{i}$ and $\rho_{i}$ were the bar length, cross section area, and density of the $i^{\text {th }}$ group bars respectively. $\sigma(A)$ is the stress constraint and $\left[\sigma_{0}\right]$ is maximum allowable stress of the group bars under various conditions, $A_{\min }$ and $A_{\max }$ are the minimum and maximum cross section area respectively.

\section{PREREQUISITE MATHEMATICS}

\subsection{Fuzzy Set}

Let $X$ is a set (space), with a generic element of $X$ denoted by $x$, that is $X(x)$.Then a Fuzzy set (FS) is defined as $A=\left\{\left(x, \mu_{A}(x)\right): x \in X\right\} \quad$ where $\quad \mu_{A}: X \rightarrow[0,1]$ is the membership function of FS $A \cdot \mu_{A}(x)$ is the degree of membership of the element $x$ to the set $A$.

\section{2 $\alpha$-Level Set or $\alpha$-cut of a Fuzzy Set}

The $\alpha$-level set of the fuzzy set $A$ of $\mathrm{X}$ is a crisp set $A_{\alpha}$ that contains all the elements of $\mathrm{X}$ that have membership values greater than or equal to $\alpha$ i.e. $A=\left\{x: \mu_{A}(x) \geq \alpha, x \in X, \alpha \in[0,1]\right\}$.

\section{3 $\alpha$-Level Set or $\alpha$-cut of a Fuzzy Set}

The $\alpha$-level set of the fuzzy set $A$ of $\mathrm{X}$ is a crisp set $A_{\alpha}$ that contains all the elements of $\mathrm{X}$ that have membership values greater than or equal to $\alpha$ i.e. $A=\left\{x: \mu_{A}(x) \geq \alpha, x \in X, \alpha \in[0,1]\right\}$.

\subsection{Convex Fuzzy Set}

A fuzzy set $A$ of the universe of discourse $X$ is convex if and only if for all $x_{1}, x_{2}$ in $X$,

$\mu_{A}\left(\lambda x_{1}+\left(1-\lambda x_{2}\right) \geq \min \left(\mu_{A}\left(x_{1}\right), \mu_{A}\left(x_{2}\right)\right)\right)$ when $0 \leq \lambda \leq 1$.

\subsection{Normal Fuzzy Set}

A fuzzy set $A$ of the universe of discourse $X$ is called a normal fuzzy set implying that there exist at least one $x \in X$ such that $\mu_{A}(x)=1$.

\section{QUASI T-NORM}

Let $T:[0,1] \times[0,1] \rightarrow[0,1]$ be a function satisfying the following axioms
a) $\quad T(a, b)=T(b, a), \forall a, b \in[0,1]$
b) $\quad T(T(a, b), c)=T(a, T(b, c)), \forall a, b, c \in[0,1]$
c) $\quad T(a, b) \leq T(a, c)$ with $b \leq c \forall a, b, c \in[0,1]$
d) $T(0,0)=0, T(1,1)=1$

\subsection{T-Norm}

A quasi-triangular norm $\mathrm{T}$ is called a triangular norm (or $\mathrm{t}$ norm) if it satisfies

$T(a, 1)=a ; \forall a \in[0,1]$

\subsection{Extended n-ary quasi-t-norms}

For the purpose of operations of multiple fuzzy sets, it is useful to define the notation of multi-dimensional t-norms. Let $[0,1]^{n}$ be an $\mathrm{n}$-dimensional cube and $\left(x_{1}, x_{2}, x_{3}, \ldots, x_{n}\right)$, $\left(z_{1}, z_{2}, \ldots ., z_{n}\right) \in[0,1]^{n}$.

A mapping $T:[0,1]^{n} \rightarrow[0,1]$ is called an n-dimensional quasi-t-norm if it satisfies the following conditions:

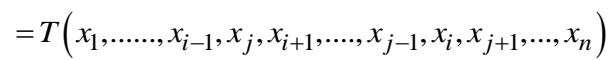

b) $\quad T\left(T\left(x_{1}, \ldots . x_{n-1}, x_{n}\right), x_{n+1}, \ldots . ., x_{2 n-1}\right)$ $=T\left(x_{1}, \ldots . . x_{n-1}, T\left(x_{n}, x_{n+1}, \ldots . ., x_{2 n-1}\right)\right)$

c) For $\quad\left(x_{1}, x_{2}, \ldots ., x_{n}\right) \leq\left(z_{1}, z_{2}, \ldots . ., z_{n}\right) \Rightarrow$ $T\left(x_{1}, x_{2}, \ldots ., x_{n}\right) \leq T\left(z_{1}, z_{2}, \ldots . ., z_{n}\right)$ with $x_{i}=z_{i}$, for some $i$ and $x_{i} \leq z_{i}$ for some $i, i=1,2,3, \ldots, n$.

d) $T(0, \ldots ., 0)=0, T(1, \ldots ., 1)=1$ 


\subsection{Extended n-ary t-norms}

An $\mathrm{n}$-dimensional quasi-t-norm $\mathrm{T}$ is called $\mathrm{n}$-dimensional $\mathrm{t}$ norm if it satisfies

$$
T\left(1,1, \ldots ., 1, x_{i}, 1, \ldots ., 1\right)=x_{i}
$$

Due to associative law it is easy to extend a triangular norm $\mathrm{T}$ into $\mathrm{n}$ arguments. The $\mathrm{n}$-ary operation $T_{n}$ on $[0,1]$ satisfies the following properties

(i) $T_{n}\left(x_{1}, x_{2}, \ldots . ., x_{n}\right)=T_{n}\left(x_{\sigma 1}, x_{\sigma 2}, \ldots . ., x_{\sigma n}\right)$ where $\sigma$ is a permutation of $\{1,2, \ldots, n\}$

(Commutativity)

(ii) $T_{n}\left(x_{1}, x_{2}, \ldots . ., x_{n}\right)$

$=T_{i+1}\left(x_{1}, x_{2}, \ldots ., x_{i}, T_{n-i}\left(x_{i+1}, \ldots . ., x_{j}, \ldots x_{n}\right)\right)$

$=T_{n-j+1}\left(T\left(x_{1}, x_{2}, \ldots ., x_{j}\right), x_{j+1}, \ldots \ldots, x_{n}\right)$

(Associativity)

(iii) $\left(\forall i \in N_{n}\right)\left(x_{i} \leq x_{i}^{\prime}\right) \Rightarrow$

$T_{n}\left(x_{1}, x_{2}, \ldots \ldots, x_{n}\right) \leq T_{n}\left(x_{1}^{\prime}, x_{2}^{\prime}, \ldots \ldots, x_{n}^{\prime}\right)$

(Monotonocity)

(iv) $T_{n}\left(x_{1}, x_{2}, \ldots ., x_{i-1}, 1, x_{i+1}, \ldots ., x_{n}\right)$

$=T\left(x_{1}, x_{2}, \ldots ., x_{i-1}, x_{i+1}, \ldots, x_{n}\right)$

(Identity law)

A t-norm $T_{n}$ is said to be continuous if $\mathrm{T}$ is continuous function on $[0,1]$. From the above, we may call $T_{n}$ is an extension of triangular norm.

\section{BASIC BINARY T-NORMS AND THEIR GENERALIZATION WITH WEIGHT FACTORS}

Minimum t-norm: $T_{M}(a, b)=\min \{a, b\}$ and extension in $\mathrm{n}$ ary of this t-norm $T_{M}\left(x_{1}, x_{2}, \ldots ., x_{n}\right)=\min \left\{x_{1}, x_{2}, \ldots \ldots x_{n}\right\}$ and extended form with weights the above t-norm $T_{M}^{W}\left(x_{1}, W_{1} ; x_{2}, W_{2} ; \ldots . ; x_{n}, w_{n}\right)=\min \left\{W_{1} x_{1}, W_{2} x_{2}, \ldots . ., W_{n} x_{n}\right\}$

Probabilistic t-norm: $T_{P}(a, b)=a . b$ and extension in n-ary of this $\quad \mathrm{t}$-norm $T_{P}\left(x_{1}, x_{2}, \ldots ., x_{n}\right)=x_{1} x_{2} \ldots \ldots . x_{n}=\prod_{i=1}^{n} x_{i}$ and extended form with weights the above t-norm $T_{P}^{W}\left(x_{1}, W_{1} ; x_{2}, W_{2} ; \ldots . . ; x_{n}, W_{n}\right)=\prod_{i=1}^{n} x_{i}^{W_{i}}$

Lukasiewicz t-norm (bounded t-norm): $T_{L}(a, b)=\max \{0, a+b-1\}$ and extension in n-ary of this $\mathrm{t}$ -

norm $\quad T_{L}\left(x_{1}, x_{2}, \ldots, x_{n}\right)=\max \left(\sum_{i=1}^{n} x_{i}-n+1,0\right)$ and extended form with weights the above t-norm $T_{L}^{W}\left(x_{1}, W_{1} ; x_{2}, W_{2} ; \ldots . . ; x_{n}, W_{n}\right)=\max \left(\sum_{i=1}^{n} W_{i} x_{i}-(n-1), 0\right)$.

\section{WEIGHTED FUZZY AGGREGATION}

Weighted Aggregation has been used quite extensively especially in fuzzy decision-making, where the weights are used to represent the relative importance the decision maker attaches to different decision criterion (goals or constraints). Weighted aggregation of fuzzy sets by using t-norms has been considered first by Yager [17]. He proposed to modify the membership functions with the associated weight factors before the fuzzy aggregation. The weighted aggregation is then the aggregation of the modified membership functions.
A general form of this idea gives the weighted aggregation function [18]

$D(x, W)=$

$$
T\left[I\left(\mu_{1}(x), W_{1}\right), I\left(\mu_{2}(x), W_{2}\right), \ldots \ldots ., I\left(\mu_{k}(x), W_{k}\right)\right]
$$

Where $W$ is a vector of weight factor $W_{i} \in[0,1] i=1,2, \ldots, k$ associated with the aggregated membership function $\mu_{i}(x)$. $T$ is t-norm and $I$ is a function of two variables that transforms the membership functions with $\sum_{i=1}^{k} W_{i}=1, W_{i} \geq 0$.

\section{MATHEMATICAL ANALYSIS}

\subsection{General Fuzzy Non-linear}

\section{Programming (FNLP) Technique to solve Multi-Objective Non-Linear Programming Problem (MONLP):}

A Multi-Objective Non-Linear Programming (MONPL) or Vector Minimization problem (VMP) may be taken in the following form:

$$
\operatorname{Min} f(x)=\left[f_{1}(x), f_{2}(x), \ldots \ldots \ldots f_{k}(x)\right]^{T}
$$

subject to

$$
\begin{aligned}
& x \in X=\left\{x \in R^{n}: g_{j}(x) \leq \text { or }=\text { or } \geq b_{j} \text { for } j=1,2,3, \ldots, m\right\} \\
& \text { and } l_{i} \leq x_{i} \leq u_{i} \quad(i=1,2,3, \ldots, n)
\end{aligned}
$$

Zimmermann (1978) showed that fuzzy programming technique can be used to solve the multi-objective programming problem.

To solve MONLP problem, following steps are used:

Step 1: Solve the MONLP (3) as a single objective non-linear programming problem using only one objective at a time and ignoring the others, these solutions are known as ideal solution.

Step 2: From the result of step 1, determine the corresponding values for every objective at each solution derived. With the values of all objectives at each ideal solution, pay-off matrix can be formulated as follows:

$$
\left.x^{1} \quad \begin{array}{cccc}
f_{1}(x) & f_{2}(x) & \cdots & f_{k}(x) \\
f_{1}^{*}\left(x^{1}\right) & f_{2}\left(x^{1}\right) & \ldots & f_{k}\left(x^{1}\right) \\
f_{1}\left(x^{2}\right) & f_{2}^{*}\left(x^{2}\right) & \ldots & f_{k}\left(x^{2}\right) \\
\ldots & \ldots & \ldots & \ldots \\
x^{k}\left(x^{k}\right) & f_{2}\left(x^{k}\right) & \ldots \ldots & f_{k}^{*}\left(x^{k}\right)
\end{array}\right)
$$

Here $x^{1} x^{2}, x^{3}, \ldots \ldots, x^{k}$ are the ideal solutions of the objectives $f_{1}(x), f_{2}(x), \ldots \ldots, f_{k}(x)$ respectively.

So $U_{r}=\max \left\{f_{r}\left(x^{1}\right), f_{r}\left(x^{2}\right), \ldots \ldots, f_{r}\left(x^{k}\right)\right\} \quad$ and $L_{r}=f_{r}^{*}\left(x^{r}\right)$ for $r=1,2, \ldots, k$ 
Where $U_{r}$ and $L_{r}$ be upper and lower bounds of the $r^{t h}$ objective function $f_{r}(x)$ for $r=1,2,3, \ldots \ldots . ., k$.

Step 3: Using aspiration level of each objective of the MONLP (3) may be written as follows:

Find $\mathrm{x}$ so as to satisfy

$$
\begin{aligned}
& f_{r}(x) \leq L_{r} \text { with tolerance } P_{r}\left(=U_{r}-L_{r}\right) \text { for } r=1,2,3, \ldots \ldots . ., k \\
& x \in X . \quad l_{i} \leq x_{i} \leq u_{i} \quad(i=1,2,3, \ldots ., n)
\end{aligned}
$$

Here objective functions of (3) are considered as fuzzy constraints. These types of fuzzy constraints can be quantified by eliciting a corresponding membership function:

$$
\begin{aligned}
\mu_{r}\left(f_{r}(x)\right)= & \begin{cases}0 & \text { if } f_{r}(x) \geq U_{r} \\
\frac{U_{r}-f_{r}(x)}{U_{r}-L_{r}} & \text { if } \quad L_{r} \leq f_{r}(x) \leq U_{r}, \\
1 \quad & \text { if } f_{r}(x) \leq L_{r}\end{cases} \\
& \text { for } r=1,2,3, \ldots, k
\end{aligned}
$$

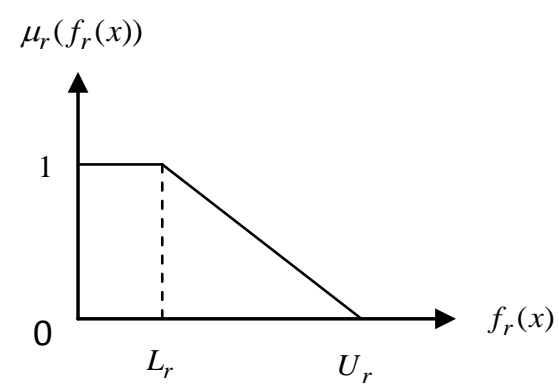

Figure 1: Membership function for objective functions

$$
f_{r}(x)
$$

After determining the different membership functions of objective functions, one can adopt following types of fuzzy decision using (2)

i) According to the extension of the weighted Zadeh's minimum t-norm operator

\section{Maximize $\alpha$}

subject to $W_{r} \mu_{r}\left(f_{r}(x)\right) \geq \alpha$,

$$
x \in X, W_{r} \geq 0, \sum_{r=1}^{k} W_{r}=1 ;
$$

ii) According to the extension of the weighted bounded tnorm operator

$$
\begin{gathered}
\text { Maximize } \mu_{D}^{A}(x ; W)=\max \left\{\sum_{r=1}^{k} W_{r} \mu_{r}\left(f_{r}(x)\right)-k+1,0\right\} \\
\text { subject to } 0 \leq \mu_{r}\left(f_{r}(x)\right) \leq 1, \\
x \in X, W_{r} \geq 0, \sum_{r=1}^{k} W_{r}=1 ;
\end{gathered}
$$

iii) According to the extension of the weighted Probabilistic t-norm operator

$$
\begin{gathered}
\text { Maximize } \mu_{D}^{P}(x ; W)=\prod_{r=1}^{k}\left(\mu_{r}\left(f_{r}(x)\right)\right)^{W_{r}} \\
\text { subject to } 0 \leq \mu_{r}\left(f_{r}(x)\right) \leq 1, \\
x \in X, W_{r} \geq 0, \sum_{r=1}^{k} W_{r}=1 ;
\end{gathered}
$$

Step 4: Solving any one among five equations (5 to 7) we will get optimal solution of (3).

\subsection{Complete Optimal Solution}

$x^{*}$ is said to be a complete optimal solution to the MONLP (3) if and only if there exists $x \in X$ such that $f_{r}\left(x^{*}\right) \leq f_{r}(x)$ for $r=1,2, \ldots, k$ and for all $x \in X$.However, when the objective functions of the MONLP conflict with each other, a complete optimal solution does not always exist and hence the Pareto Optimality Concept arises and it is defined as follows.

\subsection{Pareto Optimal Solution}

$x^{*}$ is said to be a Pareto optimal solution to the MONLP (3) if and only if there does not exist another $x \in X$ such that $f_{r}\left(x^{*}\right) \leq f_{r}(x)$ for all $r=1,2, \ldots, k$ and $f_{j}(x) \neq f_{j}\left(x^{*}\right)$ for at least one $j j \in\{1,2, \ldots, k\}$.

\section{FUZZY PROGRAMMING TECHNIQUE IN MULTI-OBJECTIVE MODEL}

To solve the above MOSOP (1), step 1 of (7.1) is used. After that according to step 2 pay-off matrix formulated as follows:

$$
\left.A^{1} \quad \begin{array}{cc}
W T(A) & \delta(A) \\
A^{2} & \delta\left(A^{1^{*}}\right) \\
W T\left(A^{1^{*}}\right) & {A^{2 *}}^{*}\left(A^{2^{*}}\right)
\end{array}\right]
$$

After that according to step 2, the bounds of objective are $U_{1}, L_{1}$ for weight function $W T(A)$ (where $L_{1} \leq W T(A) \leq U_{1}$ ) and the bounds of objective are $U_{2}, L_{2}$ for deflection function $\delta(A)$ (where $L_{2} \leq \delta(A) \leq U_{2}$ ) are identified.

Above MOSOPP reduces to a FMOSOPP as follows;

Find $A$

Such that

$$
\begin{aligned}
& W T(A) \leq L_{1} \quad \text { with maximum allowable tolerance } \\
& P_{1}\left(=U_{1}-L_{1}\right) \\
& \delta(A) \leq L_{2} \text { with maximum allowable tolerance } \\
& P_{2}\left(=U_{2}-L_{2}\right) \\
& \sigma(A) \leq\left[\sigma_{0}\right] \\
& A_{\min } \leq A \leq A_{\max }
\end{aligned}
$$


Here for simplicity linear membership functions $\mu_{W T}(W T(A))$ and $\mu_{\delta}(\delta(A))$ for the objective functions $W T(A)$ and $\delta(A)$ respectively are defined as follows;

$$
\begin{gathered}
\mu_{W T}(W T(A))= \begin{cases}1 & \text { if } W T(A) \leq L_{1} \\
\left(\frac{U_{1}-W T(A)}{U_{1}-L_{1}}\right) & \text { if } L_{1} \leq W T(A) \leq U_{1} \\
0 & \text { if } W T(A) \geq U_{1}\end{cases} \\
\mu_{\delta}(\delta(A))= \begin{cases}1 & \text { if } \delta(A) \leq L_{2} \\
\left(\frac{U_{2}-\delta(A)}{U_{2}-L_{2}^{\prime}}\right) & \text { if } L_{2} \leq \delta(A) \leq U_{2} \\
0 & \text { if } \delta(A) \geq U_{2}\end{cases}
\end{gathered}
$$

After determining the different membership functions for each of the objective functions, one can adopt following three types fuzzy decision using t-norms are

i) According to the extension of the weighted Zadeh's minimum t-norm operator

maximize $\alpha$

subject to

$$
\begin{gathered}
W_{1} \mu_{W T}(W T(A)) \geq \alpha, \quad W_{2} \mu_{\delta}(\delta(A)) \geq \alpha, \\
\sigma(A) \leq[\sigma], \\
A_{\min } \leq A \leq A_{\max }, \\
W_{1} \geq 0, W_{2} \geq 0, W_{1}+W_{2}=1 ;
\end{gathered}
$$

ii) According to the extension of the weighted bounded tnorm operator

maximize $\left(W_{1} \mu_{W T}(W T(A))+W_{2} \mu_{\delta}(\delta(A))-1,0\right)$

subject to

$$
\begin{gathered}
0 \leq \mu_{W T}(W T(A)) \leq 1, \quad 0 \leq \mu_{\delta}(\delta(A)) \leq 1, \\
\sigma(A) \leq[\sigma], \\
A_{\min } \leq A \leq A_{\max }, \\
W_{1} \geq 0, W_{2} \geq 0, W_{1}+W_{2}=1 ;
\end{gathered}
$$

iii) According to the extension of the weighted Probabilistic t-norm operator

maximize $\left(\mu_{W T}(W T(A))\right)^{W_{1}}\left(\mu_{\delta}(\delta(A))\right)^{W_{2}}$

subject to

$$
\begin{gathered}
0 \leq \mu_{W T}(W T(A)) \leq 1, \quad 0 \leq \mu_{\delta}(\delta(A)) \leq 1, \\
\sigma(A) \leq[\sigma], \\
A_{\min } \leq A \leq A_{\max }, \\
W_{1} \geq 0, W_{2} \geq 0, W_{1}+W_{2}=1 ;
\end{gathered}
$$

Solving any one among five equations (8) to (10) we will get optimal solution of (1).

\section{NUMERICAL SOLUTION OF A MULTI-OBJECTIVE STRUCTURAL OPTIMIZATION MODEL OF A THREE BAR TRUSS}

A well-known three bar [16] planar truss structure is considered. The design objective is to minimize weight of the structural $W T\left(A_{1}, A_{2}\right)$ and minimize the vertical deflection $\delta\left(A_{1}, A_{2}\right)$ at loading point of a statistically loaded three-bar planar truss subjected to stress $\sigma_{i}\left(A_{1}, A_{2}\right)$ constraints on each of the truss members $i=1,2,3$.

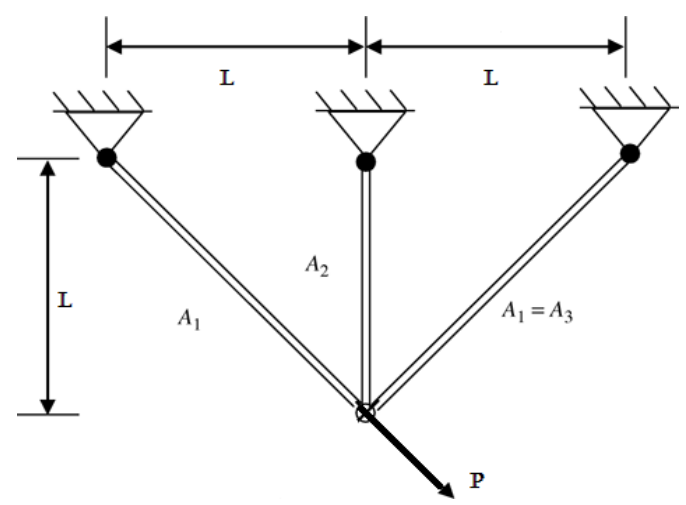

Figure 2: Design of the three-bar planar truss

The multi-objective optimization problem can be stated as follows:

$$
\begin{aligned}
& \text { minimze } W T\left(A_{1}, A_{2}\right)=\rho L\left(2 \sqrt{2} A_{1}+A_{2}\right), \\
& \text { minimize } \delta\left(A_{1}, A_{2}\right)= \frac{P L}{E\left(A_{1}+\sqrt{2} A_{2}\right)}, \\
& \text { Subject to } \sigma_{1}\left(A_{1}, A_{2}\right) \equiv \frac{P\left(\sqrt{2} A_{1}+A_{2}\right)}{2 A_{1} A_{2}+\sqrt{2} A_{1}^{2}} \leq\left[\sigma_{T}\right], \\
& \sigma_{2}\left(A_{1}, A_{2}\right) \equiv \frac{P}{A_{1}+\sqrt{2} A_{2}} \leq\left[\sigma_{T}\right], \\
& \sigma_{3}\left(A_{1}, A_{2}\right) \equiv \frac{P A_{2}}{2 A_{1} A_{2}+\sqrt{2} A_{1}^{2}} \leq\left[\sigma_{C}\right], \\
& A_{i}^{\min } \leq A_{i} \leq A_{i}^{\max }, i=1,2 .
\end{aligned}
$$

The input data for MOSOP (11) is given in table 1.

Solution: According to step 2 pay off matrix is formulated as follows;

$$
W T\left(A_{1}, A_{2}\right) \quad \delta\left(A_{1}, A_{2}\right)
$$

$$
A^{2} \quad\left[\begin{array}{ll}
2.638958 & 14.64102 \\
19.14214 & 1.656854
\end{array}\right]
$$

Here $U_{1}=19.14214, L_{1}=2.638958, U_{2}=14.64102$,

$L_{2}=1.656854$, Here linear membership function for the objective functions $W T\left(A_{1}, A_{2}\right)$ and $\delta\left(A_{1}, A_{2}\right)$ is defined as follows: 


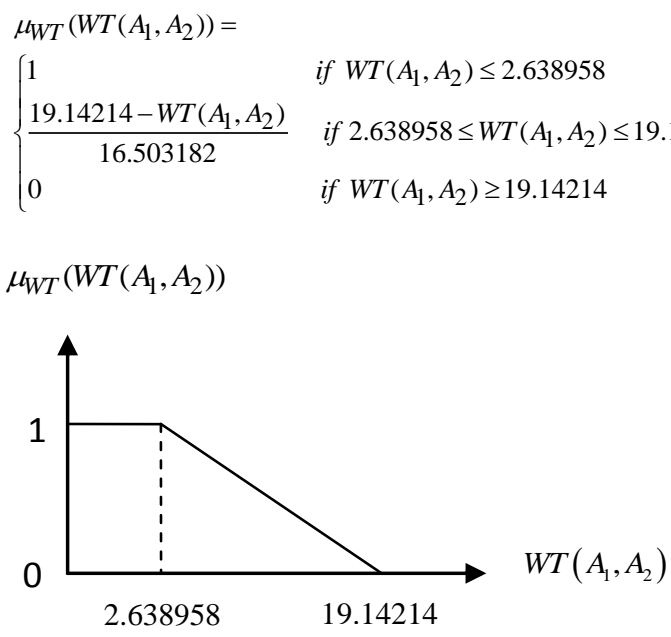

Figure 3: membership for objective weight function $W T\left(A_{1}, A_{2}\right)$

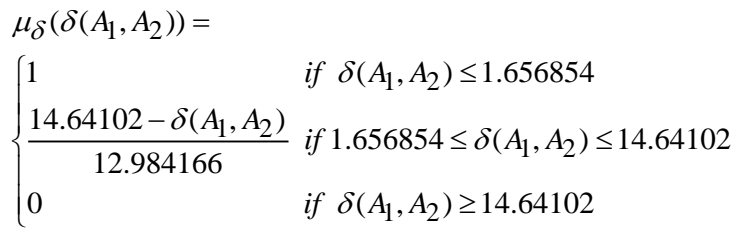

$\mu_{\delta}\left(\delta\left(A_{1}, A_{2}\right)\right)$



Figure 4: Rough sketch of membership for objective deflection functions $\delta\left(A_{1}, A_{2}\right)$

Table 1: input data for MOSOP (11) is given as follows:

\begin{tabular}{|c|c|c|c|c|c|c|}
\hline $\begin{array}{c}\text { Applied load P } \\
(K N)\end{array}$ & $\begin{array}{c}\text { Material density } \\
\rho\end{array}$ & $\begin{array}{c}\text { Length L } \\
(\mathrm{m})\end{array}$ & $\begin{array}{c}\text { Maximum } \\
\text { allowable } \\
\text { tensile } \\
\text { stress }\left[\sigma_{T}\right] \\
\left(K N / \mathrm{m}^{3}\right)\end{array}$ & $\begin{array}{c}\text { Maximum } \\
\text { allowable } \\
\text { compressive } \\
\text { stress }\left[\sigma_{C}\right] \\
\left(K N / m^{2}\right)\end{array}$ & $\begin{array}{c}\text { Young's } \\
\text { modulus } E \\
\left(K N / m^{2}\right)\end{array}$ & $\begin{array}{c}A_{i}^{\min } \text { and } A_{i}^{\text {max }} \text { of cross } \\
\text { section of bars } \\
\left(10^{-4} m^{2}\right)\end{array}$ \\
\hline 20 & 100 & 1 & 20 & 15 & $2 \times 10^{8}$ & $\begin{array}{c}A_{1}^{\min }=0.1 \\
A_{1}^{\text {max }}=5 \\
A_{2}^{\text {min }}=0.1 \\
A_{2}^{\max }=5\end{array}$ \\
\hline
\end{tabular}

Comparison of optimal solution of MOSOP (11) based on different method

Table 2: Optimal results for equal importance on Structural Weight and Deflection i.e $W_{1}=W_{2}=0.5$

\begin{tabular}{|c|c|c|c|c|}
\hline Aggregation & $A_{1} \times 10^{-4} \mathrm{~m}^{2}$ & $A_{2} \times 10^{-4} \mathrm{~m}^{2}$ & $W T\left(A_{1}, A_{2}\right) \times 10^{2} \mathrm{KN}$ & $\delta\left(A_{1}, A_{2}\right) \times 10^{-7} \mathrm{~m}$ \\
\hline Minimum & 0.5946284 & 3.470668 & 5.152531 & 3.634451 \\
\hline Bounded & 0.5995886 & 3.789756 & 5.485649 & 3.356204 \\
\hline Probabilistic & 0.5980010 & 3.682555 & 5.373957 & 3.444760 \\
\hline
\end{tabular}

For equal importance, the extension of the weighted Minimum t-norm operator gives minimum structural weight where as the extension of the weighted Bounded t-norm operator gives minimum deflection.

Table 3: Optimal results for with more importance on Structural Weight i.e $W_{1}=0.6$ and $W_{2}=0.4$

\begin{tabular}{|c|c|c|c|c|}
\hline Aggregation & $A_{1} \times 10^{-4} \mathrm{~m}^{2}$ & $A_{2} \times 10^{-4} \mathrm{~m}^{2}$ & $W T\left(A_{1}, A_{2}\right) \times 10^{2} \mathrm{KN}$ & $\delta\left(A_{1}, A_{2}\right) \times 10^{-7} \mathrm{~m}$ \\
\hline Minimum & 0.5955749 & 1.782032 & 4.528824 & 4.296216 \\
\hline Bounded & 0.5858620 & 3.003582 & 4.660650 & 4.137730 \\
\hline Probabilistic & 0.5876100 & 3.088338 & 4.750350 & 4.036181 \\
\hline
\end{tabular}

For more importance on Structural Weight, the extension of the Minimum t-norm operator gives minimum weight. 
Table 4: Optimal results for with more importance on deflection i.e $W_{1}=0.4$ and $W_{2}=0.6$

\begin{tabular}{|c|c|c|c|c|}
\hline Aggregation & $A_{1} \times 10^{-4} \mathrm{~m}^{2}$ & $A_{2} \times 10^{-4} \mathrm{~m}^{2}$ & $W T\left(A_{1}, A_{2}\right) \times 10^{2} \mathrm{KN}$ & $\delta\left(A_{1}, A_{2}\right) \times 10^{-7} m$ \\
\hline Minimum & 1.327236 & 5.000000 & 6.507334 & 2.714185 \\
\hline Bounded & 0.6111046 & 4.752674 & 6.481139 & 2.727620 \\
\hline Probabilistic & 0.6071258 & 4.377513 & 6.094724 & 2.942100 \\
\hline
\end{tabular}

For more importance on deflection, the extension of the weighted Minimum t-norm operator gives minimum deflection.

\section{CONCLUSIONS}

In this paper, we have proposed a multi-objective structural optimization model. Here binary t-norms are expressed into extended n-ary t-norms and discussed their basic properties. The said model is converted into an equivalent single objective problem and it is solved by using t-norms based fuzzy decision making technique. A main advantage of the proposed method is that it allows the user to concentrate on the actual limitations in a problem during the specification of the flexible objectives. This approximation method can be applied to optimize different models in various fields of engineering and sciences.

Conflict of interests: The authors declare that there is no conflict of interests.

\section{REFERENCES}

[1] Wang,G.Y.,Wang, W.Q.,1985, “ Fuzzy optimum design of structure.” Engineering Optimization,8,pp.291-300. .

[2] L. A. Zadeh, 1965, "Fuzzy set, Information and Control", vol.8, no.3, pp.338-353.

[3] R.E. Bellman and L.A. Zadeh,1970, “ Decision-making in a fuzzy environment, Management Science", 17(4),pp.141-164.

[4] Zimmermann, H.J.,1978, “ fuzzy linear programming with several objective function", Fuzzy sets and systems, 1 ,pp.46-55.

[5] Dey.Samir. and Roy,Tapan.Kumar., 2014,“ A Fuzzy Programming Technique for Solving Multi-objective Structural Problem", International Journal of Engineering and Manufacturing,5,pp.24-42.

[6] Xu, C. ,1989,"Fuzzy optimization of structures by the two-phase method",Computer and Structure, 31(4),pp.575-580.

[7] Yeh, Y.C, and Hsu, D.S.,1990, "Structural optimization with fuzzy parameters".Computer and Structure, 37(6), pp.917-24.

[8] C.Alsina, E. Trillas and I.Valverde,1981, "On some logical connectives for fuzzy set theory.J.Math.Anal.Appl.93,pp.15-26.

[9] Gupta,M.M., and Qi,J.,1991, "theory of t-norms and fuzzy inference methods." Fuzzy sets and systems,40,pp.431-450.

[10] Ruan, D. and Kerre,E.E.,1993, “ Fuzzy implication operators and generalized fuzzy method of cases", fuzzy sets and systems,54,pp.23-37.

[11] Pei,Z.,Pan,W., Xu,Y and Quin,K.,2003, "on some properties of fuzzy $T^{*}$-algebras and fuzzy $F^{*}$ -
algebras,IInternational Journal of

Mathematics,11,pp.7-16.

[12] Kaymak,U. and J.M. Sousa, “Weighted constraints in fuzzy optimization, ERS-2001-19-LIS

[13] Samanta, B. and Roy,T.K.,2005, "Extended t-norm and its application in portfolio selection model, Journal of fuzzy mathematics,USA,13(4),pp.995-1010.

[14] Rao, S.S., 1987, “ Description and optimum Design of Fuzzy Mathematical Systems”, Journal of Mechanisms, Transmissions, and Automation in Design, Vol.109,pp.126-132.

[15] Shih,C. J. and Lee, H. W.,2004, "Level-cut Approaches of First and Second Kind for Unique Solution Design in Fuzzy Engineering Optimization Problems", Tamkang Journal of Science and Engineering, Vol. 7, No 3, pp. 189-198.

[16] Shih,C.J., Chi,C.C. and Hsiao,J.H.,2003, "Alternative $\alpha$ -level-cuts methods for optimum structural design with fuzzy resources", Computers and Structures, 81,25792587.

[17] R. R. Yager.,1978, “ Fuzzy decision making including unequal objectives”, Fuzzy Sets and Systems,1,pp.87-95.

[18] R. R. Yager. General multiple-objective decision functions and linguistically quantified statements. International Journal of Man-Machine Studies, 21,pp.389-400, 1984.

[19] Perez, R.E. and Behdinan, K.: Particle swarm approach for structural design optimization,Computers \& Structures, Vol. 85, No. 19-20, pp. 1579-1588, 2007.

[20] Kaveh A, Talatahari S.,2010, “ An improved ant colony optimization for the design of planar steel frames", Engineering Structure, 32(3),pp.864-873.

[21] Kaveh, A., Khayatazad, M., 2013,"Ray optimization for size and shape optimization of truss structures". Computers and Structures ,117,pp.82-94.

[22] Dede T,Bekirog .lu S,Ayvaz Y.,2011, "Weight minimization of trusses with genetic algorithm". Appl Soft Comput ,11(2),pp.2565-2575.

[23] Luh GC, Lin CY.,2011, “ Optimal design of trussstructures using particle swarm optimization", Computers and Structures , 89(2324),pp.2221-2232.

[24] Sonmez M.,2011, "Discrete optimum design of truss structures using artificial bee colony algorithm". Struct Multidiscip Optimiz,43(1),pp.85-97. 